\title{
ON THE EXPECTED TIME TO RUIN AND THE EXPECTED DIVIDENDS WHEN DIVIDENDS ARE PAID WHILE THE SURPLUS IS ABOVE A CONSTANT BARRIER
}

\author{
ESTHER FROSTIG, ${ }^{*}$ University of Haifa
}

\begin{abstract}
We study the expected time to ruin in a risk process in which dividends are paid when the surplus is above the barrier. We consider the case in which the dividend rate is smaller than the premium rate. We obtain results for the classical compound Poisson risk process with phase-type claim size. When the ruin probability is 1 , we derive the expected time to ruin and the expected dividends paid. When the ruin probability is less than 1 , these quantities are derived conditioning on the event that ruin occurs.
\end{abstract}

Keywords: Risk process; ruin probability; phase-type distribution; stopping time; martingale; M/G/1 queue; busy period; idle period; $\mathrm{PH} / \mathrm{M} / 1$ queue; fluid model; change of measure; Lundberg coefficient

2000 Mathematics Subject Classification: Primary 91B30

Secondary 60K25; 60G40; 60G44

\section{Introduction}

Consider a risk process with initial reserve $u$. Claims arrive according to a Poisson process at rate $\lambda$, premium is paid at constant rate $c$, and the claim sizes are independent, identically distributed (i.i.d.) random variables $\left\{X_{i}\right\}_{i=1}^{\infty}$, with phase-type distribution with representation $(\boldsymbol{\alpha}, \boldsymbol{T})$. The surplus process $R(t)$ is defined as $R(t)=u-S(t)$, where $S(t)=\sum_{i=1}^{N(t)} X_{i}-c t$, with $S(0)=0$, and $N(t)$ is the number of arrivals up to time $t$. Let $b$ be a constant greater than $u$. When the surplus reaches the level $b$, dividends are paid at a constant rate $d$, where $d<c$. Let $c_{1}=c-d$.

Assume that $c_{1}<\lambda \mathrm{E}[X]$, that is, the ruin probability is 1 . Under this condition, we will derive the expected time to ruin and the expected dividends paid until that time. Under the assumption that $c_{1}>\lambda \mathrm{E}[X]$, that is, the ruin probability is less than 1 , we derive the ruin probability, the expected time to ruin, and the expected dividends paid until that time, given that ruin occurs.

Most of the papers that analyze risk processes with dividends assume that, when the surplus process reaches the barrier $b$, all the premium is paid as dividend, i.e. $d=c$. De Finetti (1957) was the first to suggest the use of the risk surplus process under the assumption that dividends are paid when the surplus process reaches a given barrier $b$. Bühlmann (1970) discussed various dividend policies for the surplus process in discrete time. In the case of continuous time, he presented an integrodifferential equation for the expected discounted dividends paid

Received 7 September 2004; revision received 21 December 2004.

* Postal address: Department of Statistics, University of Haifa, Haifa, 31905, Israel.

Email address: frostig@stat.haifa.ac.il 
until ruin for the fixed barrier strategy. He arrived at an explicit solution to this equation in the case in which the claim size is exponentially distributed. Gerber (1979) discussed another integrodifferential equation for the cumulative expected discounted dividends pay-out. By considering a certain martingale, he arrived at an expression for the Laplace transform of the time to ruin. Explicit results were presented only for the exponentially distributed claim size. Segerdahl (1970) discussed the ruin probability in the case of a fixed absorbing barrier, i.e. where the risk process is stopped once the barrier is reached. He found an explicit solution in the case of exponentially distributed claim size. Dickson and Gray (1984a) extended Segerdahl's (1970) results to the cases of gamma and hyperexponential claim size distributions. In another paper, Dickson and Gray (1984b) developed an alternative method of approximating the ruin probability in the Segerdahl (1970) model.

In recent years, some research has been done on analyzing probabilistic properties and deriving various quantities, such as the time to ruin and the deficit at the time to ruin, for various barrier models. Wang and Politis (2002) studied some properties of the classical risk model with a constant barrier. Having assumed that the risk process is a general random walk $S_{n}$, they found the expected time until $S_{n}$ crosses the level $b$. By applying a zero-mean martingale, they arrived at an approximation and an upper bound for the expected time at which the surplus process reaches level 0 or level $b$, and for the expected time at which the process reaches the level $b$, given that level $b$ is reached before level 0 . However, these results were obtained under the assumption that the surplus can be negative, i.e. that the risk process is not stopped when ruin occurs. Irbäck (2003) discussed the time to ruin in the classical model, assuming that the barrier $b$ is asymptotically infinite. When $u$ is close to the barrier, he proved that the time to ruin is asymptotically exponentially distributed. He also obtained the Laplace transform of the time of the first visit to the dividend barrier, given that the process reaches the barrier, and the Laplace transform of the time between two visits to the barrier. When the initial reserve $u$ is very small compared to the barrier, he found the asymptotic distribution of the time to ruin, an approximation to the probability of ruin before reaching the barrier, and the conditional probability of ruin before reaching the barrier. He also obtained an upper bound for the expected time to reach the barrier before ruin, given that this event occurs.

Lin et al. (2003), applied the well-known discounted penalty function defined by Gerber and Shiu (1998). They obtained an integral equation for the expected penalty function. The moments of the time to ruin are given by mathematical expression that are functions of the ruin probabilities in the case without barriers. Simplified expressions were given for the cases in which the claim size is either exponentially distributed or is a mixture of two exponentials. In a recent paper, Frostig (2005) derived an algorithm calculating the expected time to ruin and the expected dividends that are paid until ruin for the classical risk process with constant barrier. The author considered two models. In the first model, the claim size has a phase-type distribution. In the second model, the claim size is exponentially distributed, and the arrival rate and the mean claim size depend on a random environment. In this paper, we consider the first model, but for the case in which not all premium is paid as dividends. Another paper related to this research is the paper by Ross and Seshadri (1999), who studied the expected time until the work in an M/G/1 queueing system reaches a given level.

In Section 2, we describe the surplus process. In Section 3, we derive the expected time to ruin and the expected dividends paid until ruin occurs in the case that the ruin probability is 1 . In Section 4, we derive these quantities given that ruin occurs in the case in which the ruin probability is less than 1 . 


\section{The surplus process}

In this article, we assume that the claims are i.i.d. and distributed as is a random variable $X$, where $X$ has a phase-type distribution. Thus, $X_{j}$ is distributed in the same way as the time to absorption in a Markov process with $n$ transient states and one absorbing state, where absorption has strictly positive probability from any transient state. The distribution $G$ of $X$ is of the form

$$
G(x)=\mathrm{P}\left(X_{j} \leq x\right)=1-\alpha \mathrm{e}^{T x} \mathbf{1}
$$

for $x \geq 0$. Denote by $g$ the density function of $G$, i.e. $g(x)=\alpha \mathrm{e}^{T x} \boldsymbol{t}$. Here, $\boldsymbol{\alpha}$ is an $n$-dimensional row probability vector, $\boldsymbol{T}$ is an $n \times n$ transition rate matrix of the transient states, and $\mathbf{1}$ is an $n$-dimensional column vector of ones. By $\boldsymbol{t}=-\boldsymbol{T} \mathbf{1}$ we denote the vector of transition rates to the absorbing state. Unless stated otherwise, we assume that $c>\lambda \mathrm{E}[X]$, that is, the probability of ruin in the absence of a barrier is less than 1 . Assume that the initial reserve is a random variable $R(0)$. Let $T^{R(0)}(0, b)$ be the first time that the surplus process reaches level 0 or level $b$. Let $A_{R(0)}$ be the event that level $b$ is reached before level 0 and let $\bar{A}_{R(0)}$ be the event that ruin occurs before reaching level $b$. Let $p_{0}^{R(0)}=\mathrm{P}\left(A_{R(0)}\right)$ and let $\bar{p}^{R(0)}=1-p_{0}^{R(0)}$ be the probability of ruin before hitting the barrier, given an initial reserve $R(0)$. In the risk process without barrier, the ruin probability when the initial reserve is $u, \psi(u)$, is less than 1. By Corollary 3.1, p. 227, of Asmussen (2000), we have

$$
\psi(u)=\boldsymbol{\alpha}_{+} \mathrm{e}^{\left(\boldsymbol{T}+\boldsymbol{t} \boldsymbol{\alpha}_{+}\right) u} \mathbf{1},
$$

where $\boldsymbol{\alpha}_{+}=-(\lambda / c) \boldsymbol{\alpha} \boldsymbol{T}^{-1}$.

It was shown in Irbäck (2003) and Dickson and Gray (1984a) that, when $R(0)=u$,

$$
p_{0}^{u}=\frac{1-\psi(u)}{1-\psi(b)}
$$

and, thus,

$$
\bar{p}^{u}=\frac{\psi(u)-\psi(b)}{1-\psi(b)}
$$

Assume that the process hits $b$ at time $T^{R(0)}(0, b)$. Then the surplus process will be above the level $b$ for a random period $U_{1}$ and under the level $b$ for a random time $D_{1}$, and so on. Throughout the paper, we define the period during which the surplus process is above the level $b$ to be the period $U$, and the period during which the surplus process is below level $b$ to be the period $D$. Define a cycle as starting at the time that the surplus process crosses the level $b$ from below and ending at the first time it subsequently crosses the level $b$ from below. Denote by $C_{j}$ the (duration of the) $j$ th cycle: $C_{j}=U_{j}+D_{j}, j=1,2, \ldots$ Thus, $D_{j}$ is the time during the $j$ th cycle that the surplus is below the level $b$ and $U_{j}$ is the time during the $j$ th cycle that the surplus is above the level $b$. Note that $D_{j}, j \geq 1$, are i.i.d. random variables distributed like $D$, and that $U_{j}, j \geq 1$, are i.i.d. random variables distributed like $U$. Let $1_{j}, j \geq 1$, be indicator random variables such that $1_{j}=1$ if ruin occurs during the $j$ th cycle and $1_{j}=0$ otherwise. Note that the bivariate random vectors $\left(C_{j}, 1_{j}\right), j \geq 1$, are i.i.d. Let $N$ be the first cycle in which ruin occurs, meaning that ruin occurs during $D_{N}$. Clearly $N$ is a stopping time for the sequence $\left(\left(C_{j}, 1_{j}\right), j \geq 1\right)$. Let $1_{A}$ be the indicator of an event $A$. Denote by $B$ the 
time elapsed from the moment of ruin until the surplus process first reaches level $b$ again, let $\mathcal{T}$ be the time at which ruin occurs, and let $\mathscr{D}$ be the total dividends paid until ruin occurs. Then

$$
\begin{aligned}
& \mathcal{T}=T^{u}(0, b)+1_{A_{u}}\left(\sum_{j=1}^{N} C_{j}-B\right), \\
& \mathcal{D}=d 1_{A_{u}} \sum_{j=1}^{N} U_{j} .
\end{aligned}
$$

Similar ideas were applied in Theorem 2.2 of Ross and Seshadri (1999).

\section{The expected time to ruin and the expected dividends paid until ruin, with $c_{1}<\lambda \mathrm{E}[X]$}

Assume that

$$
c_{1}<\lambda \mathrm{E}[X] .
$$

Under this assumption, ruin occurs with probability 1 . Assume that the process hits $b$ at time $T^{u}(0, b)$. Let $p$ be the probability that ruin occurs during $D_{j}$, for some $j \geq 1$. Clearly, the stopping time $N$ is geometrically distributed with parameter $p$; thus, $\mathrm{E}[N]=1 / p$. Taking expectations in (2.3) and (2.4), and applying the Wald identity, yields

$$
\mathrm{E}[\mathcal{T}]=\mathrm{E}\left[T^{u}(0, b)\right]+p_{0}^{u}\left[\frac{\mathrm{E}[U]+\mathrm{E}[D]}{p}-\mathrm{E}[B]\right]
$$

and

$$
\mathrm{E}[D]=d p_{0}^{u} \frac{\mathrm{E}[U]}{p} .
$$

In this section, we derive $\mathrm{E}\left[T^{u}(0, b)\right], p_{0}^{R(0)}, \mathrm{E}[U], \mathrm{E}[D], p, \mathrm{E}[B]$, and, thus, $\mathrm{E}[\mathcal{T}]$ and

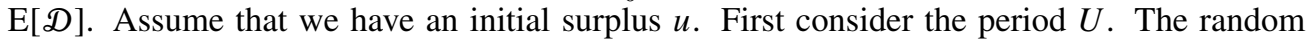
variable $U$ is distributed in the same way as the time to ruin in a risk process in which claims arrive according to a Poisson process of rate $\lambda$, the premium rate is $c_{1}$, the claim size is phasetype distributed with representation $(\boldsymbol{\alpha}, \boldsymbol{T})$, and the initial reserve is 0 . Denote this risk process by $R_{1}$. Under condition (3.1), $\mathrm{E}[U]<\infty$.

Lemma 3.1. The period $c_{1} U$ is distributed in the same way as the busy period in a $P H / M / 1$ queueing system, where the interarrival times are phase-type distributed with representation $(\boldsymbol{\alpha}, \boldsymbol{T})$ and the service times are exponentially distributed with rate $\lambda / c_{1}$.

Proof. Let $\tilde{N}$ be the number of claims that arrive until ruin occurs in the risk process $R_{1}$ described above. Let $A_{j}, j \geq 1$, be the interarrival times. Then the $A_{j}$ are independent and identically distributed as a random variable $A$, exponentially with rate $\lambda$. Thus, $\tilde{N}=\inf _{n}\left(\sum_{j=1}^{n}\left(c_{1} A_{j}-X_{j}\right)<0\right)$ and the time to ruin is $\tau_{1}=\sum_{j=1}^{N} A_{j}$. Note that $\tilde{N}$ is the number of customers that are served in a busy period $B_{1}$, where the interarrival times are distributed in the same way as $X$ and the service times are distributed in the same way as $c_{1} A$. Thus, $B_{1}=\sum_{j=1}^{N} c_{1} A_{j}$ (see Frostig (2004)).

Remark 3.1. An expression for the expected busy period in a $\mathrm{PH} / \mathrm{M} / 1$ queue (or $\mathrm{E}[U]$ ), is given on p. 111 of Neuts (1981). Let $\boldsymbol{G}$ be a matrix satisfying the equation

$$
\boldsymbol{t} \boldsymbol{\alpha} \boldsymbol{G}^{2}+\left(\boldsymbol{T}-\frac{\lambda}{c_{1}} \boldsymbol{I}\right) \boldsymbol{G}+\frac{\lambda}{c_{1}} \boldsymbol{I}=0,
$$


where $\boldsymbol{I}$ is the $n \times n$ identity matrix and $\boldsymbol{t} \boldsymbol{\alpha}$ is an $n \times n$ matrix whose $(i, j)$ th element equals $t_{i} \alpha_{j}$. Let $\boldsymbol{g}$ be the invariant vector of $\boldsymbol{G}$; that is, $\boldsymbol{g}$ is an $n$-dimensional row vector such that $\boldsymbol{g} \boldsymbol{G}=\boldsymbol{g}$ and $\boldsymbol{g} \mathbf{1}=1$. Let $\Psi^{\prime}$ be the mean of the phase-type distribution with representation $(g, T)$, i.e. $\Psi^{\prime}=-g T^{-1} 1$. Then

$$
\mathrm{E}[U]=\left(\frac{1}{1-c_{1} / \lambda \mathrm{E}[X]} \frac{\mathrm{E}\left[X^{2}\right]}{2 \mathrm{E}[X]}-\Psi^{\prime}\right) \frac{1}{c_{1}} .
$$

The overshoot below level $b$ at the end of a $U$ period is a random variable, which we denote by $Z$. Clearly, $Z$ is distributed in the same way as the deficit at ruin in the case of Poisson claims arrivals at rate $\lambda$, premium rate $c_{1}$, and phase-type distributed claim sizes with representation $(\boldsymbol{\alpha}, \boldsymbol{T})$, and has a phase-type distribution with representation $\left(\boldsymbol{\alpha}_{+}^{1}, \boldsymbol{T}\right)$ (see Proposition 4.1, p. 229, of Asmussen (2000), and see below for the definition of $\boldsymbol{\alpha}_{+}^{1}$ ). Note that $Z=\sum_{j=1}^{N}\left(X_{j}-c_{1} A_{j}\right)$. This is exactly the expression for the idle period in a $\mathrm{G} / \mathrm{M} / 1$ queueing system, as described in Remark 3.1 (see Frostig (2004)). Let $\theta$ be the least positive root of the equation

$$
r=\mathrm{E}\left[\mathrm{e}^{-\left(\lambda / c_{1}\right)(1-r) X}\right] .
$$

It can be shown that $\theta<1$ if and only if $\left(\lambda / c_{1}\right) \mathrm{E}[X]>1$, and that $\theta=1$ otherwise. The idle period has a density given by (see Section 8.1, p. 35, of Prabhu (1997b))

$$
\begin{aligned}
f_{Z}(x) & =\frac{\lambda}{c_{1}} \int_{x}^{\infty} \exp \left(-\frac{\lambda}{c_{1}}(1-\theta)(y-x)\right) g(y) \mathrm{d} y \\
& =\frac{\lambda}{c_{1}} \int_{x}^{\infty} \exp \left(-\frac{\lambda}{c_{1}}(1-\theta)(y-x)\right) \boldsymbol{\alpha} \mathrm{e}^{\boldsymbol{T} y} \boldsymbol{t} \mathrm{d} y \\
& =-\frac{\lambda}{c_{1}} \boldsymbol{\alpha}\left(\boldsymbol{T}-\frac{\lambda}{c_{1}}(1-\theta) \boldsymbol{I}\right)^{-1} \mathrm{e}^{\boldsymbol{T} x} \boldsymbol{t} .
\end{aligned}
$$

That is, $Z$ has a phase-type distribution with representation $\left(\boldsymbol{\alpha}_{+}^{1}, \boldsymbol{T}\right)$, where

$$
\boldsymbol{\alpha}_{+}^{1}=-\frac{\lambda}{c_{1}} \boldsymbol{\alpha}\left(\boldsymbol{T}-\frac{\lambda}{c_{1}}(1-\theta) \boldsymbol{I}\right)^{-1} .
$$

Remark 3.2. Note that if $\left(\lambda / c_{1}\right) \mathrm{E}[X]<1$, then $\theta=1$. In this case, $\boldsymbol{\alpha}_{+}^{1}=-\left(\lambda / c_{1}\right) \boldsymbol{\alpha} \boldsymbol{T}^{-1}$ and $\alpha_{1 j}^{+}$is the probability that ruin occurs and the claim hits level 0 during phase $j$. This result is derived in Section 3a, p. 227, of Asmussen (2000) by applying different arguments.

Thus,

$$
\begin{aligned}
f_{Z}(z) & =\boldsymbol{\alpha}_{+}^{1} \mathrm{e}^{\boldsymbol{T} z} \boldsymbol{t}, \\
\mathrm{P}(Z>z) & =\boldsymbol{\alpha}_{+}^{1} \mathrm{e}^{\boldsymbol{T} z} \mathbf{1}, \\
\mathrm{E}[Z] & =-\boldsymbol{\alpha}_{+}^{1} \boldsymbol{T}^{-1} \mathbf{1} .
\end{aligned}
$$

Lemma 3.2. The mean of a $D$ period is given by

$$
\mathrm{E}[D]=\frac{\boldsymbol{\alpha}_{+}^{1} \boldsymbol{T}^{-1} \mathbf{1}}{c-\lambda \mathrm{E}[X]} .
$$




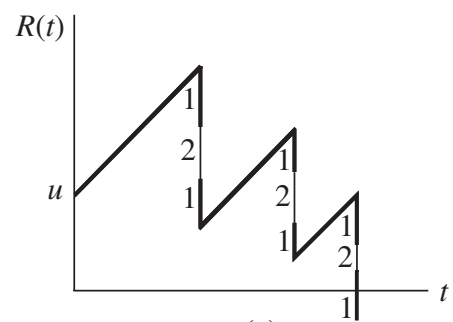

(a)

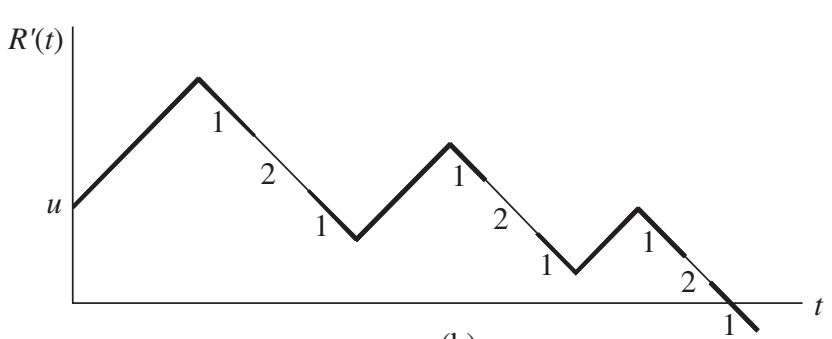

(b)

FIGURE 1: The process $R(t)$ and its fluid representation $R^{\prime}(t)$.

Proof. The $D$ period is distributed in the same way as the busy period of an M/G/1 queue with Poisson arrivals at rate $\lambda$, where the service time of the first customer in the busy period is distributed in the same way as $Z$, the service time of a customer that arrives during the busy period is distributed in the same way as $X$, and the service rate is equal to $c$. Thus, (3.4) follows from a known result for M/G/1 queues (see p. 138 of Prabhu (1997a)) and (3.3).

Next, we derive the probability $p$ of ruin during a $D$ period. If $Z>b$ then ruin occurs at the end of the $U$ period. Given that $Z=z<b$, the probability that ruin occurs before reaching level $b$ again is

$$
\bar{p}^{b-z}=\frac{\psi(b-z)-\psi(b)}{1-\psi(b)}
$$

(see (2.2)). Thus,

$$
p=\mathrm{P}(Z>b)+\int_{0}^{b} \frac{\psi(b-x)-\psi(b)}{1-\psi(b)} f_{Z}(x) \mathrm{d} x,
$$

where $\psi(u)$ is as given in (2.1).

Consider the surplus process $R(t)$ and assume that $0<R(0)<b$. Note that $R(t)$ increases at rate $c$ and has phase-type-distributed downwards jumps. When a downwards jump crosses the level 0 (ruin occurs) it can be in one of the phases $1, \ldots, n$. In Figure 1(a), we illustrate the process $R(t)$, where the claims are phase-type distributed with two phases denoted by 1 and 2. Let $p_{j}^{R(0)}, j=1, \ldots, n$, be the probability that ruin occurs before reaching level $b$ and during claim phase $j$, let $p_{0}^{R(0)}$ be the probability of reaching $b$ before ruin, and let $p^{R(0)}$ be the $(n+1)$-dimensional probability vector $\boldsymbol{p}^{R(0)}=\left(p_{0}^{R(0)}, \ldots, p_{n}^{R(0)}\right)$.

To find $\boldsymbol{p}^{R(0)}$, we apply the theory of Markov additive processes. In order to do so, we first present the fluid description of the surplus process, denoted by $R^{\prime}(t)$. The process $R^{\prime}(t)$ increases at rate $c$ until a claim arrives. If the claim is of size $v$ then the process starts to decrease at rate 1 for $v$ time units and then increases again at rate $c$; that is, in the fluid model, the downwards jumps are (pictorially speaking) replaced by segments with slope -1 . This description was also used by Asmussen and Kella (2000) and Kella et al. (2003). In Figure 1(b), we illustrate the fluid representation of $R(t)$.

The fluid representation enables us to apply the multidimensional martingale for Markov additive process introduced in Asmussen and Kella (2000). The process $R^{\prime}(t)$ is a Markov additive process in which the modulating states are $0,1, \ldots, n$. When in state 0 , the process increases at rate $c$ and, when in states $1, \ldots, n$, the process decreases at rate 1 .

Let $J_{t}$ be the modulating state of $R^{\prime}(t)$ at time $t$. Note that the probability that $R^{\prime}(t)$ hits level $b$ before level 0 , starting with initial surplus $R(0)$, is $p_{0}^{R(0)}$, and the probability that it hits 
level 0 when the modulating state is $j$ is $p_{j}^{R(0)}, j=1, \ldots, n$. The process $\left\{J_{t}, t \geq 0\right\}$ is a Markov process with a generator $\boldsymbol{Q}$, where

$$
Q=\left(\begin{array}{cc}
-\lambda & \lambda \alpha \\
t & T
\end{array}\right)
$$

Let $\boldsymbol{F}_{t}(\gamma)$ be a matrix with $(i, j)$ th element $\mathrm{E}_{i}\left[\mathrm{e}^{\gamma R^{\prime}(t)} ; J_{t}=j\right]$ (where, for a random variable $X$ and an event $A, \mathrm{E}[X ; A]:=\mathrm{P}(A) \mathrm{E}[X \mid A])$. Then $\boldsymbol{F}_{t}(\gamma)=\mathrm{e}^{t \boldsymbol{K}(\gamma)}$, where

$$
\boldsymbol{K}(\gamma)=\boldsymbol{Q}+\left(\begin{array}{cc}
c \gamma & \mathbf{0} \\
\mathbf{0} & -\gamma \boldsymbol{I}
\end{array}\right)
$$

(see Proposition 2.2, p. 311, of Asmussen (2003)). The matrix $K(\gamma)$ has a real eigenvalue $\kappa(\gamma)$ with maximal real part (see p. 312 of Asmussen (2003)). Let $\boldsymbol{h}^{\gamma}=\left(h_{0}^{\gamma}, \ldots, h_{n}^{\gamma}\right)^{\top}$ be its corresponding right eigenvector. By Proposition 2.3, p. 312, of Asmussen (2003), $\mathrm{e}^{R^{\prime}(t)-t \kappa(\gamma)} h_{J_{t}}^{\gamma}$ is a martingale. Assume that the equation $\operatorname{det} \boldsymbol{K}(\gamma)=0$ has $n+1$ roots, $\gamma_{0}, \ldots, \gamma_{n}$. Let $\boldsymbol{h}^{j}, j=0, \ldots, n$ be the eigenvector corresponding to $\gamma_{j}$. Note that one of the roots is 0 and that the components of the corresponding eigenvector are all equal to 1 . Thus (see Proposition 2.4, p. 312, of Asmussen (2003) and Lemma 2.1 of Asmussen and Kella (2000)), $\mathrm{e}^{\gamma_{j} R^{\prime}(t)} h_{J_{t}}^{j}$ is a martingale. Let $T^{\prime R(0)}(0, b)$ be the first time that $R^{\prime}(t)$ exits $[0, b]$, starting at $R(0)$. Note that $J_{T^{\prime}(0)}(0, b)=0$ if $R^{\prime}\left(T^{\prime R(0)}(0, b)\right)=b$, and that $J_{0}=0$. Thus, an application of the optional sampling theorem yields

$$
\mathrm{E}\left[\mathrm{e}^{\gamma_{j} R(0)} h_{0}^{j}\right]=\mathrm{e}^{b} p_{0}^{R(0)} h_{0}^{j}+\sum_{k=1}^{n} p_{k}^{R(0)} h_{k}^{j} .
$$

Thus, we obtain $n+1$ equations for $p_{0}^{R(0)}, \ldots, p_{n}^{R(0)}$.

Remark 3.3. (i) Note that the probability of ruin before reaching the barrier $b$ is

$$
\bar{p}^{R(0)}=\sum_{k=1}^{n} p_{k}^{R(0)} .
$$

(ii) Given that ruin occurs before the surplus reaches level $b$, the deficit at ruin has a phase-type distribution with representation $\left(\tilde{\boldsymbol{p}}^{R(0)}, \boldsymbol{T}\right)$, where $\tilde{\boldsymbol{p}}^{R(0)}=\left(p_{1}^{R(0)} / \bar{p}^{R(0)}, \ldots, p_{n}^{R(0)} / \bar{p}^{R(0)}\right)$.

Lemma 3.3. The mean of $T^{u}(0, b)$ is given by

$$
\mathrm{E}\left[T^{u}(0, b)\right]=\frac{b p_{0}^{u}+\bar{p}^{u} \tilde{\boldsymbol{p}}^{u} \boldsymbol{T}^{-1} \mathbf{1}}{c-\lambda \mathrm{E}[X]} .
$$

Proof. Given that ruin occurs, the deficit at ruin has a phase-type distribution with representation $\left(\tilde{\boldsymbol{p}}^{R(0)}, \boldsymbol{T}\right)$ and mean $-\tilde{\boldsymbol{p}}^{R(0)} \boldsymbol{T}^{-1} \mathbf{1}$. The process $R(t)-(c-\lambda \mathrm{E}[X]) t$ is a zero-mean martingale. An application of the optional sampling theorem yields

$$
\mathrm{E}\left[R\left(T^{u}(0, b)\right)\right]-(c-\lambda \mathrm{E}[X]) \mathrm{E}\left[T^{u}(0, b)\right]=0 .
$$

Thus,

$$
b p_{0}^{u}+\bar{p}^{u} \tilde{\boldsymbol{p}}^{u} \boldsymbol{T}^{-1} \mathbf{1}-(c-\lambda \mathrm{E}[X]) \mathrm{E}\left[T^{u}(0, b)\right]=0
$$

and the result follows. 
We now derive $\mathrm{E}[B]$, the time elapsed between the moment that the surplus downcrosses level 0 and the moment that it reaches level $b$.

Proposition 3.1. Let $\Delta$ be the deficit at ruin when ruin occurs during a $D$ period. Then

$$
\mathrm{E}[B]=\frac{b+\mathrm{E}[\Delta]}{c-\lambda \mathrm{E}[X]} .
$$

Proof. The time elapsed before the surplus process reaches the barrier $b$ is distributed in the same way as a busy period in an M/G/1 queueing system with Poisson arrivals at rate $\lambda$, where the service time of a customer that arrives during the busy period is distributed in the same way as $X$, the service time of the first customer in the busy period is distributed in the same way as $b+\Delta$, and the service rate is $c$. Thus, the result follows (see p. 138 of Prabhu (1997a)).

To find $\mathrm{E}[\Delta]$, we consider two cases: $Z>b$ and $Z \leq b$. First let $Z>b$. Then (3.3) implies that the overshoot, or the deficit at ruin, $\Delta$, is phase-type distributed with representation $\left(\boldsymbol{\alpha}_{+}^{1} \mathrm{e}^{\boldsymbol{T} b}, \boldsymbol{T}\right)$. Thus,

$$
\mathrm{E}[\Delta ; Z>b]=-\boldsymbol{\alpha}_{+}^{1} \mathrm{e}^{\boldsymbol{T} b} \boldsymbol{T}^{-1} \mathbf{1} .
$$

Now let $Z \leq b$. Then, given that ruin occurs, $\Delta$ is phase-type distributed with representation $\left(\tilde{\boldsymbol{p}}^{b-Z_{b}}, \boldsymbol{T}\right)$, where $Z_{b}$ is distributed in the same way as the conditional distribution of $Z$ given that $Z \leq b$. We derive $\tilde{\boldsymbol{p}}^{b-Z_{b}}$ from (3.5) and Remark 3.3(ii) by substituting for the distribution of $R(0)$ with the distribution of $b-Z_{b}$. Thus, the conditional distribution of $\Delta$ given that $Z \leq b$ is

$$
\mathrm{E}[\Delta \mid Z \leq b]=-\bar{p}^{b-Z_{b}} \tilde{\boldsymbol{p}}^{b-Z_{b}} \boldsymbol{T}^{-1} \mathbf{1}
$$

Corollary 3.1. The mean of $B$ is given by

$$
\mathrm{E}[B]=\frac{b}{c-\lambda \mathrm{E}[X]}-\frac{\boldsymbol{\alpha}_{+}^{1} \mathrm{e}^{\boldsymbol{T} b} \boldsymbol{T}^{-1} \mathbf{1}+\mathrm{P}(Z \leq b) \bar{p}^{b-Z_{b}} \tilde{\boldsymbol{p}}^{b-Z_{b}} \boldsymbol{T}^{-1} \mathbf{1}}{c-\lambda \mathrm{E}[X]} .
$$

\section{The expected time to ruin and the expected dividends paid until ruin, with $c_{1}>\lambda \mathrm{E}[X]$}

In the case in which $c_{1}>\lambda \mathrm{E}[X]$, the probability of ruin is less than 1 . In this case, we will first find the ruin probability and then the expected time to ruin and the expected dividends paid, given that ruin occurs.

\subsection{The ruin probability}

Starting with initial surplus $u$, ruin occurs before reaching level $b$ with probability $\bar{p}^{u}$, as in (2.2). With probability $p_{0}^{u}$, the surplus reaches level $b$ before ruin. Given that $A_{u}$ occurs, ruin occurs if and only if $N<\infty$. In other words, ruin occurs if there exists an $n<\infty$ such that each period $U_{j}$ is finite for $j=1, \ldots, n$, and occurs during $D_{n}$. In this case, $N=n$. Let $C_{U}$ be the event of ruin for a risk process with Poisson arrivals at rate $\lambda$, claim size distributed in the same way as $X$, premium rate $c_{1}$, and initial reserve 0 . The probability that a $U$ period is finite is the same as the probability of $C_{U}$ :

$$
\mathrm{P}\left(C_{U}\right)=: \psi_{1}=\frac{\lambda \mathrm{E}[X]}{c_{1}} .
$$


Let $\tilde{Z}$ be the deficit at the end of the $U$ period, i.e. the deficit when ruin occurs. Given that $U$ is finite (ruin occurs), $\tilde{Z}$ has the following density function and expectation (see Theorem 12.2, p. 103, of Bowers et al. (1986)):

$$
\begin{aligned}
f_{\tilde{Z}}(z) & =\frac{1-G(z)}{\mathrm{E}[X]}, \\
\mathrm{E}[\tilde{Z}] & =\frac{\mathrm{E}\left[X^{2}\right]}{2 \mathrm{E}[X]} .
\end{aligned}
$$

Remark 4.1. Let $\boldsymbol{v}=\boldsymbol{\alpha}_{1}^{+} c / \lambda \mathrm{E}[X]$, where $\boldsymbol{\alpha}_{1}^{+}$is as defined in Remark 3.2. Then, given that the $U$ period is finite, $\tilde{Z}$ has a phase-type distribution with representation $(\boldsymbol{v}, \boldsymbol{T})$, where $\boldsymbol{v}=-\boldsymbol{\alpha} \boldsymbol{T}^{-1} / \mathrm{E}[X]$ (see Corollary 2.3, p. 224, of Asmussen (2000)).

Let $C_{D}$ be the event of ruin occurring during a $D$ period. Given $C_{U}$, the probability $p$ of ruin during a $D$ period is

$$
\begin{aligned}
p & =\mathrm{P}(\tilde{Z}>b)+\int_{0}^{b} \frac{\psi(b-x)-\psi(b)}{1-\psi(b)} f_{\tilde{Z}}(x) \mathrm{d} x \\
& =\boldsymbol{v e}^{\boldsymbol{T} b} \mathbf{1}+\int_{0}^{b} \frac{\psi(b-x)-\psi(b)}{1-\psi(b)} \boldsymbol{v e}^{\boldsymbol{T} x} \boldsymbol{t} \mathrm{d} x,
\end{aligned}
$$

where $\psi(u)$ is as in (2.1). Thus, the ruin probability is

$$
\mathrm{P}(\mathcal{T}<\infty)=\bar{p}^{u}+p_{0}^{u} \Psi
$$

where

$$
\Psi=\psi_{1} p \sum_{j=0}^{\infty}\left(\psi_{1}(1-p)\right)^{j}=\frac{\psi_{1} p}{1-\psi_{1}(1-p)} .
$$

\subsection{The expected time to ruin and the expected dividends paid given that ruin occurs}

First we assume that the initial reserve is $b$; that is, dividends are paid from time 0 until the surplus process downcrosses the barrier $b$. Let the time to ruin be $\mathcal{T}_{b}$, and let $\mathscr{D}_{b}$ be the total dividends paid until ruin. The event $\left(\mathcal{T}_{b}<\infty\right)$ occurs if and only if the event $(N<\infty) \cap\left(U_{j}<\infty, D_{j}<\infty, j=1, \ldots, N\right)$ occurs. Thus,

$$
\begin{aligned}
\mathrm{E}\left[\mathcal{T}_{b} ; \mathcal{T}_{b}<\infty\right]= & \mathrm{E}\left[\sum_{j=1}^{N}\left(U_{j}+D_{j}\right) 1\left(N<\infty, U_{j}<\infty, D_{j}<\infty, j=1, \ldots, N\right)\right] \\
& -\mathrm{E}\left[B ; \mathcal{T}_{b}<\infty\right] .
\end{aligned}
$$

Proposition 4.1. When the time to ruin is finite, its expectation is

$$
\begin{aligned}
\mathrm{E}\left[\mathcal{T}_{b} ; \mathcal{T}_{b}<\infty\right]= & \mathrm{E}\left[\sum_{j=1}^{N}\left(U_{j}+D_{j}\right) 1\left(N<\infty, U_{j}<\infty, D_{j}<\infty, j=1, \ldots, N\right)\right] \\
& -\mathrm{E}\left[B ; \mathcal{T}_{b}<\infty\right] \\
= & \mathrm{E}[U+D ; U<\infty, D<\infty] \mathrm{E}[N ; N<\infty] \\
& -\mathrm{E}\left[B \mid \mathcal{T}_{b}<\infty\right] \mathrm{P}\left(\mathcal{T}_{b}<\infty\right) .
\end{aligned}
$$


Proof. The proof is the same as the proof of the Wald identity (see, e.g. Theorem 3.3.3, p. 59, of Ross (1983)).

To find $\mathrm{E}[U ; U<\infty]$, we apply the change-of-measure theorem. Let $M_{X}(r)=\mathrm{E}\left[\mathrm{e}^{r X}\right]$ be the moment-generating function of $X$ at $r$. Let $\gamma$ be the Lundberg coefficient, i.e. the positive solution to the equation $\lambda M_{X}(r)-c_{1} r-\lambda=0$, let

$$
S_{1}(t)=\sum_{j=1}^{N(t)} X_{j}-c_{1} t
$$

let $\tau_{1}=\inf \left\{t: S_{1}(t)>0\right\}$, and let $\left\{\mathscr{F}_{t}\right\}_{t \geq 0}$ be the natural filtration adapted to $S_{1}(t)$. With respect to $\left\{\mathcal{F}_{t}\right\}_{t \geq 0}$, the process $L_{\gamma}(t)=\mathrm{e}^{\gamma \bar{S}_{1}(t)}$ is a martingale with mean 1 . For $A \in \mathcal{F}_{t}$, let

$$
\mathrm{P}_{\gamma}(A)=\mathrm{E}\left[L_{\gamma}(t) ; A\right] .
$$

By Proposition 2.2, p. 27, of Asmussen (2000), or Proposition 3.1, p. 359, of Asmussen (2003), $\mathrm{P}_{\gamma}$ is a well-defined probability measure.

The probability measure $\mathrm{P}_{\gamma}$ defines a risk process $R_{\gamma}(t)=-S_{\gamma}(t)$ with the following parameters: claims arrive according to a Poisson process at rate $\lambda_{\gamma}=\lambda M_{X}(\gamma)$, the premium rate is $c_{1}$, and the claim sizes are distributed in the same way as a random variable $X_{\gamma}$, with density function

$$
g_{\gamma}(x)=\frac{\mathrm{e}^{\gamma x} g(x)}{M_{X}(\gamma)} .
$$

Let $\boldsymbol{h}=(-\gamma \boldsymbol{I}-\boldsymbol{T})^{-1} \boldsymbol{t}$, and let $\boldsymbol{\Delta}$ be a diagonal matrix with the elements of $\boldsymbol{h}$ on the diagonal. Then $X_{\gamma}$ has a phase-type distribution with representation $\left(\boldsymbol{\alpha}_{\gamma}, \boldsymbol{T}_{\gamma}\right)$, where

$$
\begin{aligned}
& \boldsymbol{T}_{\gamma}=\boldsymbol{\Delta}^{-1} \boldsymbol{T} \boldsymbol{\Delta}+\gamma \boldsymbol{I}, \\
& \boldsymbol{\alpha}_{\gamma}=\frac{\boldsymbol{\alpha} \boldsymbol{\Delta}}{M_{X}(\gamma)} .
\end{aligned}
$$

The corresponding exit rate vector is $\boldsymbol{t}_{\gamma}=\boldsymbol{\Delta}^{-1} \boldsymbol{t}$ (see, e.g. Asmussen (1989), (1992)).

In the risk process $R_{\gamma}$, ruin occurs with probability 1 (see p. 362 of Asmussen (2003)). Thus, under the measure $\mathrm{P}_{\gamma}, U$ is distributed in the same way as the busy period in a $\mathrm{PH} / \mathrm{M} / 1$ queueing system in which the interarrival times are phase-type distributed with representation $\left(\boldsymbol{\alpha}_{\gamma}, \boldsymbol{T}_{\gamma}\right)$ and the service times are exponentially distributed with rate $\lambda_{\gamma}$. Denote by $\mathrm{E}_{\gamma}$ the expectation operator with respect to $\mathrm{P}_{\gamma}$. As in (3.2), let $\boldsymbol{G}_{\gamma}$ be a matrix satisfying the equation

$$
\boldsymbol{t}_{\gamma} \boldsymbol{\alpha}_{\gamma} \boldsymbol{G}_{\gamma}^{2}+\left(\boldsymbol{T}_{\gamma}-\frac{\lambda_{\gamma}}{c_{1}} \boldsymbol{I}\right) \boldsymbol{G}_{\gamma}+\frac{\lambda}{c_{1}} \boldsymbol{I}=0 .
$$

Let $\boldsymbol{B}_{\gamma}$ be an $n \times n$ matrix solving the equation

$$
\boldsymbol{B}_{\gamma}=-\left(\boldsymbol{T}_{\gamma}-\lambda_{\gamma} \boldsymbol{I}\right)^{-1} \boldsymbol{G}_{\gamma}-\left(\boldsymbol{T}_{\gamma}-\lambda_{\gamma} \boldsymbol{I}\right)^{-1} \boldsymbol{t}_{\gamma} \boldsymbol{\alpha}_{\gamma}\left(\boldsymbol{G}_{\gamma} \boldsymbol{B}_{\gamma}+\boldsymbol{B}_{\gamma} \boldsymbol{G}_{\gamma}\right)
$$

The $(i, j)$ th component of $\boldsymbol{B}_{\gamma}$ is the expected busy period of a $\mathrm{PH} / \mathrm{M} / 1$ queue starting at arrival phase $i$ and terminating at arrival phase $j$. Thus, the $j$ th component of the vector $\boldsymbol{\alpha}_{\gamma} \boldsymbol{B}_{\gamma}$ equals the busy period starting at phase $i$ with probability $\alpha_{\gamma, i}, i=1, \ldots, n$, and ending at arrival phase $j$ (see Equation (3.3.20), p. 101, of Neuts (1981)). 
Proposition 4.2. When a $U$ period is finite, its expectation is

$$
\mathrm{E}[U ; U<\infty]=\boldsymbol{\alpha}_{\gamma} \boldsymbol{B}_{\gamma}\left(\gamma \boldsymbol{I}-\boldsymbol{T}_{\gamma}\right)^{-1} \boldsymbol{t}_{\gamma} .
$$

Proof. We have

$$
\mathrm{E}[U ; U<\infty]=\mathrm{E}_{\gamma}\left[\mathrm{e}^{-\gamma S_{1}(U)} U\right],
$$

by Theorem 2.3, p. 27, of Asmussen (2000) or Theorem 3.3, p. 359, of Asmussen (2003). Note that $S_{1}(U)$ is the deficit when ruin occurs, and that, under the probability measure $\mathrm{P}_{\gamma}$, it has a phase-type distribution with representation $\left(\boldsymbol{\beta}, \boldsymbol{T}_{\gamma}\right)$, where $\boldsymbol{\beta}$ is the vector of probabilities that the surplus process hits level 0 at phase $j, j=1, \ldots, n$. The Laplace transform of $S_{1}(U)$ is $\boldsymbol{\beta}\left(s \boldsymbol{I}-\boldsymbol{T}_{\gamma}\right)^{-1} \boldsymbol{t}_{\gamma}$. According to the discussion just before Proposition 4.2, the $j$ th component of $\boldsymbol{\alpha}_{\gamma} \boldsymbol{B}_{\gamma}$ is the expected value of the $U$ period starting at phase $i$ with probability $\alpha_{\gamma, i}$ and terminating at phase $j$. Given that the $U$ period ends at phase $j, j=1, \ldots, n$, the deficit $S_{1}(U)$ is independent of the length of the $U$ period. Thus, the result follows.

We now derive $\mathrm{E}[D ; D<\infty]$. Given that $U<\infty, D$ is distributed in the same way as the busy period in an M/G/1 queueing system in which the service time of the first customer is distributed as in (4.2), the service times of all the other customers arriving during the busy period are distributed in the same way as $X$, and the service rate is $c$. The expectation of such a busy period is

$$
\mathrm{E}[D \mid U<\infty]=\frac{\mathrm{E}\left[X^{2}\right]}{2 \mathrm{E}[X](c-\lambda \mathrm{E}[X])} .
$$

Thus,

$$
\mathrm{E}[D ; U<\infty]=\psi_{1} \frac{\mathrm{E}\left[X^{2}\right]}{2 \mathrm{E}[X](c-\lambda \mathrm{E}[X])},
$$

where $\psi_{1}$ is as given in (4.1). Let $\Delta$ be the deficit at ruin, given that ruin occurs. To find $E[\Delta]$ we consider two cases: $\tilde{Z}>b$ and $\tilde{Z} \leq b$. First let $\tilde{Z}>b$. In this case, by Remark $4.1, \Delta$ has a phase-type distribution with representation $\left(\boldsymbol{v} \mathrm{e}^{T b}, \boldsymbol{T}\right)$ and, thus,

$$
\mathrm{E}[\Delta ; \tilde{Z}>b]=-v \mathrm{e}^{T b} \boldsymbol{T}^{-1} \mathbf{1} .
$$

Now let $\tilde{Z} \leq b$. Let $\tilde{Z}_{b}$ be a random variable distributed in the same way as $(\tilde{Z} \mid \tilde{Z} \leq b)$. Given that $\tilde{Z} \leq b, \Delta$ has a phase-type distribution with representation

$$
\left(\bar{p}^{b-\tilde{Z}_{b}} \tilde{\boldsymbol{p}}^{b-\tilde{Z}_{b}}, \boldsymbol{T}\right),
$$

where $\tilde{\boldsymbol{p}}^{b-\tilde{Z}_{b}}$ can be derived from Remark 3.3 and (3.5).

Proposition 4.3. Given that ruin occurs, the expected time $\mathrm{E}\left[B \mid \mathcal{T}_{b}<\infty\right]$ from that point until level $b$ is reached again is

$$
\mathrm{E}\left[B \mid \mathcal{T}_{b}<\infty\right]=\frac{b}{c-\lambda \mathrm{E}[X]}-\frac{\boldsymbol{v e}^{\boldsymbol{T} b} \boldsymbol{T}^{-1} \mathbf{1}+\mathrm{P}(\tilde{Z} \leq b) \bar{p}^{b-\tilde{Z}_{b}} \tilde{\boldsymbol{p}}^{b-\tilde{Z}_{b}} \boldsymbol{T}^{-1} \mathbf{1}}{c-\lambda \mathrm{E}[X]} .
$$

Proof. The time elapsed between the moment that ruin occurs and the moment that the surplus again reaches the level $b$ is distributed in the same way as the busy period in an M/G/1 queueing system that has a Poisson arrival process of rate $\lambda$, a service time distribution $G$, a service rate $c_{1}$, and which is such that the service time of the first customer in the busy period is distributed in the same way as $b+\Delta$. Thus, the result follows. 
The following proposition is now straightforward.

Proposition 4.4. The mean values of $\mathcal{T}_{b}$ and $\mathscr{D}_{b}$, given that $\mathcal{T}_{b}$ is finite, are

$$
\begin{aligned}
\mathrm{E}\left[\mathcal{T}_{b} \mid \mathcal{T}_{b}<\infty\right]= & \frac{1}{\Psi}(\mathrm{E}[U ; U<\infty]+\mathrm{E}[D ; D<\infty]) \frac{\psi_{1} p}{\left(1-\psi_{1}(1-p)\right)^{2}} \\
& -\mathrm{E}\left[B \mid \mathcal{T}_{b}<\infty\right], \\
\mathrm{E}\left[\mathscr{D}_{b} \mid \mathcal{T}_{b}<\infty\right]= & \frac{\mathrm{E}[U ; U<\infty]}{\Psi} \frac{\psi_{1} p}{\left(1-\psi_{1}(1-p)\right)^{2}} .
\end{aligned}
$$

Next, assume that the initial reserve is $u, 0<u<b$. Then

$$
\begin{aligned}
\mathrm{E}[\mathcal{T} ; \mathcal{T}<\infty]= & \bar{p}^{u} \mathrm{E}\left[T^{u}(0, b) \mid R\left(T^{u}(0, b)\right)<0\right] \\
& +p_{0}^{u}\left(\mathrm{E}\left[T^{u}(0, b) \mid R\left(T^{u}(0, b)\right)=b\right] 1\left(\mathcal{T}_{b}<\infty\right)+\mathrm{E}\left[\mathcal{T}_{b} ; \mathcal{T}_{b}<\infty\right]\right) \\
\leq & \bar{p}^{u} \mathrm{E}\left[T^{u}(0, b) \mid R\left(T^{u}(0, b)\right)<0\right] \\
& +p_{0}^{u}\left(\mathrm{E}\left[T^{u}(0, b) \mid R\left(T^{u}(0, b)\right)=b\right]+\mathrm{E}\left[\mathcal{T}_{b} ; \mathcal{T}_{b}<\infty\right]\right) \\
= & \mathrm{E}\left[T^{u}(0, b)\right]+p_{0}^{u} \mathrm{E}\left[\mathcal{T}_{b} ; \mathcal{T}_{b}<\infty\right]
\end{aligned}
$$

and, thus,

$$
\begin{aligned}
& \mathrm{E}[\mathcal{T} \mid \mathcal{T}<\infty] \leq \frac{\mathrm{E}\left[T^{u}(0, b)\right]+p_{0}^{u} \mathrm{E}\left[\mathcal{T}_{b} ; \mathcal{T}_{b}<\infty\right]}{\mathrm{P}(\mathcal{T}<\infty)}, \\
& \mathrm{E}[\mathcal{D} \mid \mathcal{T}<\infty]=\frac{p_{0}^{u} \mathrm{E}\left[\mathscr{D}_{b} \mid \mathcal{T}_{b}<\infty\right] \Psi}{\mathrm{P}(\mathcal{T}<\infty)} .
\end{aligned}
$$

\section{References}

Asmussen, S. (1989). Exponential families generated by phase-type distributions and other Markov lifetimes. Scand. J. Statist. 16, 319-334.

Asmussen, S. (1992). Phase-type representations in random walk and queueing problems. Ann. Appl. Prob. 20, 772-789.

Asmussen, S. (2000). Ruin Probabilities (Adv. Ser. Statist. Sci. Appl. Prob. 2). World Scientific, River Edge, NJ.

Asmussen, S. (2003). Applied Probability and Queues (Appl. Math. (New York) 51), 2nd edn. Springer, New York.

Asmussen, S. And Kella, O. (2000). A multi-dimensional martingale for Markov additive processes and its applications. Adv. Appl. Prob. 32, 376-393.

Bowers, N. L. et al. (1986). Actuarial Mathematics. Society of Actuaries, Schaumburg, IL.

BüHlmann, H. (1970). Mathematical Methods in Risk Theory. Springer, Berlin.

De FinetTi, B. (1957). Su un impostazione alternativa della teoria collectiva del rischio. Trans. XV Internat. Cong. Actuaries 2, 433-443.

Dickson, D. C. M And Gray, J. R. (1984a). Approximations to ruin probability in the presence of an upper absorbing barrier. Scand. Actuarial J. 1984, 105-115.

Dickson, D. C. M. AND GRAY, J. R. (1984b). Exact solutions for ruin probability in the presence of an absorbing upper barrier. Scand. Actuarial J. 1984, 174-186.

Frostig, E. (2004). Upper bounds on the expected time to ruin and on the expected recovery time. Adv. Appl. Prob. 36, 377-397.

Frostig, E. (2005). The expected time to ruin in a risk process with constant barrier via martingale. To appear in Insurance Math. Econom.

Gerber, H. U. (1979). An Introduction to Mathematical Risk Theory (S. S. Heubner Foundation Monogr. Ser. 8). Irwin, Homewood, IL.

Gerber, H. U. And ShiU, E. S. W. (1998). On the time value of ruin. North Amer. Actuarial J. 2, 48-78.

IRBÄCK, J. (2003). Asymptotic theory for a risk process with high dividend barrier. Scand. Actuarial J. 2003, 97-118.

Kella, O., Perry, D. and Stadje, W. (2003). A stochastic clearing model with a Brownian and a compound Poisson components. Prob. Eng. Inf. Sci. 17, 1-22.

Lin, X. S., Willmot, G. E. AND Drekic, S. (2003). The classical risk model with constant dividend barrier: analysis of the Gerber-Shiu discounted penalty function. Insurance Math. Econom. 33, 551-566. 
Neuts, M. F. (1981). Matrix-Geometric Solutions in Stochastic Models. An Algorithmic Approach (Johns Hopkins Ser. Math. Sci. 2). Johns Hopkins University Press, Baltimore, MD.

Prabhu, N. U. (1997a). Foundations of Queueing Theory. Kluwer, Boston, MA.

Prabhu, N. U. (1997b). Stochastic Storage Processes. Queues, Insurance Risk, Dams, and Data Communication (Appl. Math. (New York) 15). Springer, New York.

Ross, S. M. (1983). Stochastic Processes. John Wiley, New York.

Ross, S. M. AND SeShadri, S. (1999). Hitting time in an M/G/1 queue. J. Appl. Prob. 36, 934-940.

SEGERDAHL, C.-O. (1970). On some distributions in time connected with the collective theory of risk. Scand. Actuarial J. 1970, 167-192.

WANG, N. AND Politis, K. (2002). Some characteristics of a surplus process in the presence of an upper barrier. Insurance Math. Econom. 30, 231-241. 